\title{
A RECURRENCE FORMULA FOR THE SOLUTIONS OF CERTAIN LINEAR PARTIAL DIFFERENTIAL EQUATIONS
}

\section{MORRIS MARDEN}

1. Introduction. In a number of recent papers, Bergman ${ }^{1}$ has developed the theory of operational methods for transforming analytic functions of a complex variable into solutions of the linear partial differential equation

$$
L(U)=U_{z \bar{z}}+a(z, \bar{z}) U_{z}+b(z, \bar{z}) U_{\bar{z}}+c(z, \bar{z}) U=0,
$$

where $z=x+i y, \bar{z}=x-i y$,

$$
\begin{gathered}
U_{z}=\frac{1}{2}\left(\frac{\partial U}{\partial x}-i \frac{\partial U}{\partial y}\right), \quad U_{z}=\frac{1}{2}\left(\frac{\partial U}{\partial x}+i \frac{\partial U}{\partial y}\right), \\
U_{z z}=\frac{1}{4}\left(\frac{\partial^{2} U}{\partial x^{2}}+\frac{\partial^{2} U}{\partial y^{2}}\right)=\frac{\Delta U}{4},
\end{gathered}
$$

and where the coefficients $a(z, \bar{z}), b(z, \bar{z})$ and $c(z, \bar{z})$ are analytic functions of both variables $z$ and $\bar{z}$. The equation (1.1) is equivalent to the system of two real equations

$$
\begin{gathered}
\Delta U^{(1)}+2 A U_{x}^{(1)}+2 B U_{y}^{(1)}+2 C U_{x}^{(2)}+2 D U_{y}^{(2)} \\
+4 c_{1} U^{(1)}-4 c_{2} U^{(2)}=0, \\
\Delta U^{(2)}-2 C U_{x}^{(1)}-2 D U_{y}^{(1)}+2 A U_{x}^{(2)}+2 B U_{y}^{(2)} \\
+4 c_{2} U^{(1)}+4 c_{1} U^{(2)}=0,
\end{gathered}
$$

where

$$
\begin{array}{lll}
U=U^{(1)}+i U^{(2)} ; & 2 A=(a+\bar{a})+(b+\bar{b}) ; & 2 B=i[(\bar{a}-a)-(\bar{b}-b)] ; \\
c=c_{1}+i c_{2} ; & 2 D=(a+\bar{a})-(b+\bar{b}) ; & 2 C=i[(a-\bar{a})+(b-\bar{b})] .
\end{array}
$$

Presented to the Society, November 26, 1943; received by the editors November 13, 1943. This paper was prepared while the author was a fellow under the Program of Advanced Instruction in Mechanics at Brown University.

'S. Bergman, (a) Zur Theorie der Funktionen, die eine lineare partielle Differentialgleichung befriedigen, Rec. Math. (Mat. Sbornik) N.S. vol. 44 (1937) pp. 1169-1198; (b) The approximation of functions satisfying a linear partial differential equation, Duke Math. J. vol. 6 (1940) pp. 537-561; (c) Linear operators in the theory of partial differential equations, Trans. Amer. Math. Soc. vol. 53 (1943) pp. 130-155; (d) On the solutions of partial differential equations of the fourth order, to appear later. 
Furthermore, if $a=b$ and $c$ is real, then $C=D=c_{2}=0$, and the two differential equations become real and identical.

For equations (1.1), Bergman proved the existence of two functions $E_{1}(z, \bar{z}, t)$ and $E_{2}(z, \bar{z}, t)$, called by him "generating functions of the first kind," 2 with the following properties:

(1) They have the forms

$$
\begin{aligned}
& E_{1}(z, \bar{z}, t)=\exp \left(-\int_{0}^{\bar{z}} a(z, \bar{z}) d \bar{z}\right)\left[1+z \bar{z} t E_{1}^{*}(z, \bar{z}, t)\right], \\
& E_{2}(z, \bar{z}, t)=\exp \left(-\int_{0}^{z} b(z, \bar{z}) d z\right)\left[1+z \bar{z} t E_{2}^{*}(z, \bar{z}, t)\right],
\end{aligned}
$$

where each $E_{k}^{*}(z, \bar{z}, t)$ has continuous first partial derivatives in $z, \bar{z}$ and $t$ for $|t| \leqq 1$ and for $z$ and $\bar{z}$ within a certain four-dimensional region.

(2) The classes $C\left(E_{1}\right)$ and $C\left(E_{2}\right)$ of functions $U_{1}(z, \bar{z})$ and $U_{2}(z, \bar{z})$ defined by the formulas

$$
\begin{aligned}
& U_{1}(z, \bar{z})=\int_{-1}^{1} E_{1}(z, \bar{z}, t) f\left(z\left(1-t^{2}\right) / 2\right) d t /\left(1-t^{2}\right)^{1 / 2}, \\
& U_{2}(z, \bar{z})=\int_{-1}^{1} E_{2}(z, \bar{z}, t) g\left(\bar{z}\left(1-t^{2}\right) / 2\right) d t /\left(1-t^{2}\right)^{1 / 2},
\end{aligned}
$$

where $f(\zeta)$ and $g(\zeta)$ are arbitrary analytic functions of $\zeta$, form subsets of solutions of (1.1).

(3) Every solution $U(z, \bar{z})$ of (1.1) may be written in the form

$$
U(z, \bar{z})=U_{1}(z, \bar{z})+U_{2}(z, \bar{z}),
$$

with $f(\zeta)$ and $g(\zeta)$ suitably chosen analytic functions.

As was proved by Bergman, to many theorems about analytic functions of a complex variable correspond analogous theorems about functions belonging to classes $C(E)$ generated by functions $E$ of the first kind. In particular, if we define as "basic solutions" those corresponding to $f(z)=z^{p}$, that is

$$
u_{p}(z, \bar{z})=\int_{-1}^{1} E(z, \bar{z}, t)\left[z\left(1-t^{2}\right) / 2\right]^{p} d t /\left(1-t^{2}\right)^{-1 / 2},
$$

then every function $U$ of class $C(E)$ which is regular in $|z| \leqq r$ may

2 Generating functions which are considered as not of the first kind are those failing to satisfy property (1). When $E$ is a generating function not of the first kind, the integration in (1.2) and (1.3) must be taken along a rectifiable curve joining the points $t= \pm 1$, but not passing through $t=0$. 
be expanded in a series $U=\sum \alpha_{p} u_{p}$ which is uniformly and absolutely convergent in $|z| \leqq r$.

For example, in the case of the equation

$$
\Delta U+U=0,
$$

$E(z, \bar{z}, t)=e^{i t r}$, where $z=r e^{i \theta}$ and thus $r=(z \bar{z})^{1 / 2}$. Because of the well known formula for the Bessel function of the first kind

the basic solutions are

$$
J_{p}(r)=(2 / \pi)(1 / \Gamma(p+1 / 2)) \int_{-1}^{1} e^{r t i}(r / 2)^{p}\left(1-t^{2}\right)^{p-1 / 2} d t,
$$

$$
u_{p}(r, \theta)=\left(\pi^{1 / 2} / 2\right) \Gamma(p+1 / 2) e^{p \theta i} J_{p}(r) .
$$

But for (1.5), successive terms in the expansion $U=\sum \alpha_{p} u_{p}$ can be computed from earlier terms by the use of some recurrence relation satisfied by the Bessel's functions, as for example the relation

$$
J_{p}^{\prime}(r)=(p / r) J_{p}(r)-J_{p+1}(r) .
$$

It would likewise be of practical value in the case of other differential equations $L(U)=0$ to determine what recurrence relations, if any, are satisfied by the basic solutions $u_{p}(r, \theta)$.

In the present note, recurrence formulas connecting the basic solutions $u_{p}(r, \theta)$ are found in the case of differential equations $L(U)=0$ for which at least one of the corresponding "generating functions" $E(z, \bar{z}, t)$ is of the form $E(z, \bar{z}, t)=\exp f(r, \theta, t)$ where $f(r, \theta, t)$ is a polynomial in $t$ containing either only even powers of $t$ or only odd powers of $t$. Obviously, the equation (1.5) is an example of such an equation. Other examples can be found by requiring the coefficients $a, b$ and $c$ in the equation $L(U)=0$ to satisfy certain differential relations. ${ }^{3}$

Our first main result may be stated as follows:

THEOREM 1. Let $L(U)=0$ be a partial differential equation of the type (1.1) for which there exists a generating function having one of the forms:

$$
\begin{aligned}
& E(z, \bar{z}, t)=\exp P(r, \theta, t), \\
& E(z, \bar{z}, t)=\exp t P(r, \theta, t),
\end{aligned}
$$

where $P(r, \theta, t)=a_{0}(r, \theta)+a_{1}(r, \theta) t^{2}+\cdots+a_{n}(r, \theta) t^{2 n}$, and where the coefficients $a_{j}(r, \theta)$ are of class $C^{\prime}$ in $r$ and $\theta$. Let $u_{p}(r, \theta)$ be the corresponding "basic solutions" of equation $L(U)=0$ and let

${ }^{3}$ See reference in footnote 1a, pp. 1194-1195, and also p. 158 of the following article: K. L. Nielsen and B. P. Ramsay, On particular solutions of linear partial differential equations, Bull. Amer. Math. Soc. vol. 49 (1943) pp. 156-162. 


$$
\begin{aligned}
& \alpha_{q}(r, \theta)=(-1)^{q}\left[\sum_{j=q}^{n} C_{j, q} \frac{\partial a_{j}}{\partial r}\right]\left(\frac{2}{r e^{i \theta}}\right)^{q}, \\
& \beta_{q}(r, \theta)=(-1)^{q}\left[\sum_{j=q}^{n}(2 j+1) C_{j, q} a_{j}\right]\left(\frac{2}{r e^{i \theta}}\right)^{q} .
\end{aligned}
$$

Then, if $E$ has form (I),

$$
\frac{\partial u_{p}}{\partial r}=\frac{p}{r} u_{p}+\sum_{j=0}^{n} \alpha_{j} u_{p+j} ;
$$

whereas, if $E$ has form (II),

$$
\frac{\partial u_{p}}{\partial r}=\frac{p}{r} u_{p}+\frac{2}{r e^{i \theta}} \sum_{j, k=0}^{n} \frac{\alpha_{j} \beta_{k} u_{j+k+p+1}}{2 j+2 p+1} .
$$

The above theorem will be derived as an immediate consequence of two lemmas that are given in the next section. In the third section the theorem will be applied to a few specific equations of form (1.1).

2. Two lemmas. First we shall derive a result for polynomials $P(r, \theta, t)$ involving only even powers of $t$.

LEMMA 1. Let

$$
P(r, \theta, t)=a_{0}(r, \theta)+a_{1}(r, \theta) t^{2}+\cdots+a_{n}(r, \theta) t^{2 n},
$$

where the $a_{j}(r, \theta)$ are functions of class $C^{\prime}$ in $r$ and $\theta$. Then the function

$$
u_{p}(r, \theta)=\int_{-1}^{1} e^{P(r, \theta, t)}\left(1-t^{2}\right)^{p-1 / 2}\left(r e^{i \theta} / 2\right)^{p} d t
$$

satisfies the recurrence formula

$$
\partial u_{p} / \partial r=\left[p / r+P_{r}\left(r, \theta,(1-T)^{1 / 2}\right)\right] u_{p},
$$

where $T$ is the operator such that, $T$ acting $k$ times upon $u_{p}$,

$$
T^{k} u_{p}=\left(2 / r e^{i \theta}\right)^{k} u_{p+k}
$$

for $k=0,1, \cdots$.

Proof. From (2.1) we obtain by differentiating with respect to $r$ :

$$
\begin{aligned}
\frac{\partial u_{p}}{\partial r}=\frac{p}{r} u_{p}+\int_{-1}^{1} e^{P}\left[\frac{\partial a_{0}}{\partial r}+\right. & \frac{\partial a_{1}}{\partial r} t^{2}+\cdots \\
& \left.+\frac{\partial a_{n}}{\partial r} t^{2 n}\right]\left(1-t^{2}\right)^{p-1 / 2}\left(\frac{r e^{i \theta}}{2}\right) d t .
\end{aligned}
$$


To evaluate the latter integral, let us note that

$$
\begin{aligned}
\int_{-1}^{1} t^{2 m} e^{P}\left(1-t^{2}\right)^{p-1 / 2} & \left(r e^{i \theta} / 2\right)^{p} d t \\
& =\int_{-1}^{1}\left[1-\left(1-t^{2}\right)\right]^{m} e^{P}\left(1-t^{2}\right)^{p-1 / 2}\left(r e^{i \theta} / 2\right)^{p} d t \\
& =\sum_{k=0}^{m}(-1)^{k} C_{m, k} \int_{-1}^{1} e^{P}\left(1-t^{2}\right)^{k+p-1 / 2}\left(r e^{i \theta} / 2\right)^{p} d t \\
& =\sum_{k=0}^{m}(-1)^{k} C_{m, k}\left(2 / r e^{i \theta}\right)^{p} u_{p+k} .
\end{aligned}
$$

Hence,

$$
\int_{-1}^{1} t^{2 m} e^{P}\left(1-t^{2}\right)^{p-1 / 2}\left(r e^{i \theta} / 2\right)^{p} d t=(1-T)^{m} u_{p}
$$

Substituting now from (2.5) into (2.4), we find

$$
\begin{aligned}
\frac{\partial u_{p}}{\partial r} & =\frac{p}{r} u_{p}+\frac{\partial a_{0}}{\partial r} u_{p}+\frac{\partial a_{1}}{\partial r}(1-T) u_{p}+\cdots+\frac{\partial a_{n}}{\partial r}(1-T)^{n} u_{p} \\
& =\left[p / r+P_{r}\left(r, \theta,(1-T)^{1 / 2}\right)\right] u_{p},
\end{aligned}
$$

as was to be proved.

The corresponding result for a polynomial that involves only odd powers of $t$ may be stated as follows.

LEMMA 2. Let $Q(r, \theta, t) \equiv a_{0}(r, \theta) t+a_{1}(r, \theta) t^{3}+\cdots+a_{n}(r, \theta) t^{2 n+1}$ $\equiv t P(r, \theta, t)$, where the $a_{j}(r, \theta)$ are functians of class $C^{\prime}$ in $r$ and $\theta$. Then the functions $u_{p}(r, \theta)$ defined by (2.1) satisfy the recurrence formula:

$$
\begin{aligned}
\partial u_{p} / \partial r=(p / r) u_{p}+ & \left\{Q_{t}\left(r, \theta,(1-T)^{1 / 2}\right)\right. \\
& \left.\cdot \int_{0}^{T^{1 / 2}} t^{2 p} P_{r}\left(r, \theta,(1-t)^{1 / 2}\right) d t\right\} T^{-p+1 / 2} u_{p},
\end{aligned}
$$

where $T$ is the operator defined by equation (2.3).

Proof. In place of (2.4), we now have

$$
\begin{aligned}
\frac{\partial u_{p}}{\partial r}=\frac{p}{r} u_{p}+\int_{-1}^{1} e^{Q}\left[\frac{\partial a_{0}}{\partial r}\right. & t+\frac{\partial a_{1}}{\partial r} t^{3}+\cdots \\
& \left.+\frac{\partial a_{n}}{\partial r} t^{2 n+1}\right]\left(1-t^{2}\right)^{p-1 / 2}\left(\frac{r e^{i \theta}}{2}\right)^{p} d t
\end{aligned}
$$

In order to evaluate the latter integral, let us first integrate by parts: 


$$
\begin{aligned}
\int_{-1}^{1} t e^{Q}(1 & \left.-t^{2}\right)^{p-1 / 2}\left(r e^{i \theta} / 2\right)^{p} d t \\
& =-\frac{1}{2} \int_{-1}^{1} e^{Q}\left(1-t^{2}\right)^{p-1 / 2}\left(r e^{i \theta} / 2\right)^{p} d\left(1-t^{2}\right) \\
& =(1 /(2 p+1)) \int_{-1}^{1} e^{Q}(\partial Q / \partial t)\left(1-t^{2}\right)^{p+1 / 2}\left(r e^{i \theta} / 2\right)^{p} d t \\
& =(1 /(2 p+1)) \int_{-1}^{1} e^{Q}\left[a_{0}+3 a_{1} t^{2}+\cdots\right. \\
& \left.\quad+(2 n+1) a_{n} t^{2 n}\right]\left(1-t^{2}\right)^{p+1 / 2}\left(r e^{i \theta} / 2\right)^{p} d t .
\end{aligned}
$$

Hence, by equation (2.5),

$$
\begin{array}{r}
\int_{-1}^{1} t e^{Q}\left(1-t^{2}\right)^{p-1 / 2}\left(r e^{i \theta} / 2\right)^{p} d t=(1 /(2 p+1)) Q_{t}\left(r, \theta,(1-T)^{1 / 2}\right) T u_{p} \\
=Q_{t}\left(r, \theta,(1-T)^{1 / 2}\right)\left(\int_{0}^{T^{1 / 2}} t^{2 p} d t\right) T^{-p+1 / 2} u_{p} .
\end{array}
$$

Let us then assume that the formula

$$
\begin{aligned}
& \int_{-1}^{1} t^{2 m+1} e^{Q}\left(1-t^{2}\right)^{p-1 / 2}\left(r e^{i \theta} / 2\right)^{p} d t \\
& \quad=Q_{t}\left(r, \theta,(1-T)^{1 / 2}\right)\left(\int_{0}^{T^{1 / 2}} t^{2 p}\left(1-t^{2}\right)^{m} d t\right) T^{-p+1 / 2} u^{p}
\end{aligned}
$$

has already been verified for $m=0,1,2, \cdots, N$ and proceed to verify the formula for $m=N+1$, as follows.

$$
\begin{aligned}
\int_{-1}^{1} t^{2 N+3} e^{Q}(1 & \left.-t^{2}\right)^{p-1 / 2}\left(r e^{i \theta} / 2\right)^{p} d t \\
= & \int_{-1}^{1} t^{2 N+1} e^{Q}\left(1-t^{2}\right)^{p-1 / 2}\left(r e^{i \theta} / 2\right)^{p} d t \\
& -\int_{-1}^{1} t^{2 N+1} e^{Q}\left(1-t^{2}\right)^{p+1 / 2}\left(r e^{i \theta / 2}\right)^{p} d t \\
= & Q_{t}\left(r, \theta,(1-T)^{1 / 2}\right)\left\{\left[\int_{0}^{T^{1 / 2}} t^{2 p}\left(1-t^{2}\right)^{N} d t\right] T^{-p+1 / 2} u_{p}\right. \\
& \left.-\left[\int_{0}^{T^{1 / 2}} t^{2 p+2}\left(1-t^{2}\right)^{N} d t\right]\left(2 / r e^{i \theta}\right) T^{-p-1 / 2} u_{p+1}\right\} \\
= & Q_{t}\left(r, \theta,(1-T)^{1 / 2}\right)\left(\int_{0}^{T^{1 / 2}} t^{2 p}\left(1-t^{2}\right)^{N+1} d t\right) T^{-p+1 / 2} u_{p} .
\end{aligned}
$$


Thus, formula (2.8) has been established by mathematical induction.

We now substitute from formula (2.8) into expression (2.7), thus obtaining

$$
\begin{aligned}
\frac{\partial u_{p}}{\partial r}= & \frac{p}{r} u_{p}+\left(Q_{t}\left(r, \theta,(1-T)^{1 / 2}\right)\right. \\
& \left.\cdot \int_{0}^{T^{1 / 2}} t^{2 p}\left\{\frac{\partial a_{0}}{\partial r}+\frac{\partial a_{1}}{\partial r}\left(1-t^{2}\right)+\cdots+\frac{\partial a_{n}}{\partial r}\left(1-t^{2}\right)^{n}\right\}\right) T^{-p+1 / 2} u_{p} \\
= & \frac{p}{r} u_{p}+Q_{t}\left(r, \theta,(1-T)^{1 / 2}\right)\left\{\int_{0}^{T^{1 / 2}} t^{2 p} P_{r}\left(r, \theta,(1-t)^{1 / 2}\right)\right\} T^{-p+1 / 2} u_{p},
\end{aligned}
$$

as was to be proved.

Proof of Theorem 1. Formula (2.2) may be reduced to formula (1.10) if, using (1.8) and (2.3), we set

$$
\begin{aligned}
P_{r}\left(r, \theta,(1-T)^{1 / 2}\right) u_{p} & =\sum_{j=0}^{n} \frac{\partial a_{j}}{\partial r}(1-T)^{i} u_{p}=\sum_{q=0}^{n} \alpha_{q}\left(\frac{r e^{i \theta}}{2}\right)^{q} T^{q} u_{p} \\
& =\sum_{q=0}^{n} \alpha_{q} u_{p+q} .
\end{aligned}
$$

To reduce formula (2.6) to (1.11), let us set

$$
Q_{t}\left(r, \theta,(1-T)^{1 / 2}\right)=\sum_{j=0}^{n}(2 j+1) a_{j}(1-T)^{i}=\sum_{j=0}^{n} \beta_{j}\left(\frac{r e^{i \theta}}{2}\right)^{j} T^{j}
$$

with the $\beta_{j}$ defined as in formula (1.9). Since

$$
P_{r}\left(r, \theta,\left(1-t^{2}\right)^{1 / 2}\right)=\sum_{j=0}^{n} \alpha_{j}\left(\frac{r e^{i \theta}}{2}\right)^{i} t^{2 i}
$$

we may write the second term of the left side of (2.6) as

$$
\begin{aligned}
& \sum_{k=0}^{n} \beta_{k}\left(\frac{r e^{i \theta}}{2}\right)^{k} T^{k}\left[\int_{0}^{T^{1 / 2}} t^{2 p} \sum_{j=0}^{n} \alpha_{j}\left(\frac{r e^{i \theta}}{2}\right)^{j} t^{2 j} d t\right] T^{-p+1 / 2} u_{p} \\
& =\sum_{j, k=0}^{n} \frac{\alpha_{j} \beta_{k}}{2 p+2 j+1}\left(\frac{r e^{i \theta}}{2}\right)^{i+k} T^{k+j+1} u_{p}=\frac{2}{r e^{i \theta}} \sum_{j, k=0}^{n} \frac{\alpha_{j} \beta_{k} u_{p+j+k+1}}{2 p+2 j+1} .
\end{aligned}
$$

Thus the proof of our main theorem is completed.

3. Examples. Let us first verify that the recurrence relation (2.6) is a generalization of that for Bessel's functions as given in formula (1.7). Here $Q(r, \theta, t)=r t i$ and thus (2.6) becomes 


$$
\begin{aligned}
\partial u_{p} / \partial r & =(p / r) u_{p}+r i\left[\int_{0}^{T^{1 / 2}} t^{2 p} i d t\right] T^{-p+1 / 2} u_{p} \\
& \left.=(p / r) u_{p}-\left(2 e^{-i \theta /(2 p}+1\right)\right) u_{p+1} .
\end{aligned}
$$

If now we set

$$
\begin{aligned}
u_{p} & =\left(\pi^{1 / 2} / 2\right) \Gamma(p+1 / 2) J_{p}(r) e^{i p \theta}, \\
u_{p+1} & =\left(\pi^{1 / 2} / 2\right)(p+1 / 2) \Gamma(p+1 / 2) J_{p+1}(r) e^{i(p+1) \theta},
\end{aligned}
$$

formula (3.1) reduces at once to formula (1.7).

As our second example, let us consider the differential equation $L(U)=0$ in which the expression $F=c-a b-a_{z} \neq 0$ satisfies the two equations

$$
F_{z}=0, \quad 2 F-a_{z}+b_{z}=0 .
$$

As shown by Bergman, ${ }^{4}$ one of the possible corresponding generating functions is $E(z, \bar{z}, t)=\exp P(r, \theta, t)=\exp \left(a_{0}+a_{1} t^{2}\right)$, where

$$
a_{0}=-\int_{0}^{\bar{z}} a d \bar{z}, \quad a_{1}=2 z \int_{0}^{\bar{z}} F d \bar{z} .
$$

According to our theorem, the recurrence relation satisfied by the basic solutions is in this case

$$
\left(\partial u_{p} / \partial r\right)=(p / r) u_{p}+\alpha_{0} u_{p}+\alpha_{1} u_{p+1}
$$

where

$$
\begin{aligned}
& \alpha_{0}=\partial a_{0} / \partial r+\partial a_{1} / \partial r=-a e^{-i \theta}+2 r F+2 e^{i \theta} \int_{0}^{z} F d \bar{z}, \\
& \alpha_{1}=-\left(2 / r e^{i \theta}\right)\left(\partial a_{1} / \partial r\right)=-4 e^{-i \theta} F-(4 / r) \int_{0}^{z} F d \bar{z} .
\end{aligned}
$$

A partial differential equation which satisfies conditions (3.2) is

$$
U_{z \bar{z}}-2(z+\bar{z}) U_{\bar{z}}+U=0 .
$$

Here $F(z)=1, a_{0}=0, a_{1}=2 r^{2}$, and therefore in the recurrence relation (3.4) $\alpha_{0}=4 r$, and $\alpha_{1}=-8 e^{-i \theta}$. Setting $U=U^{(1)}+i U^{(2)}$, we see that equation (3.5) is equivalent to the system of two partial differential equations

$$
\begin{aligned}
& \Delta U^{(1)}-8 x U_{x}^{(1)}+8 x U_{y}^{(2)}+4 U^{(1)}=0, \\
& \Delta U^{(2)}-8 x U_{x}^{(2)}-8 x U_{y}^{(1)}+4 U^{(2)}=0,
\end{aligned}
$$

- See p. 1194, reference in footnote 1 . 
and that recurrence relation (3.4) for $u_{p}=u_{p}^{(1)}+i u_{p}^{(2)}$ is in this case equivalent to the system of recurrence relations

$$
\begin{aligned}
& \partial u_{p}^{(1)} / \partial r=(p / r) u_{p}^{(1)}+4 r u_{p}^{(1)}-8 u_{p+1}^{(1)} \cos \theta-8 u_{p+1}^{(2)} \sin \theta, \\
& \partial u_{p}^{(2)} / \partial r=(p / r) u_{p}^{(2)}+4 r u_{p}^{(2)}-8 u_{p+1}^{(2)} \cos \theta+8 u_{p+1}^{(1)} \sin \theta .
\end{aligned}
$$

Other examples of partial differential equations $L(U)=0$ for which $\log E$ is an even or odd polynomial in $t$ may be found in the articles referred to in footnotes $1 \mathrm{a}$ and 3 . For these differential equations also, a recurrence relation may be derived by use of Lemmas 1 and 2 .

4. Generalization. By means of formulas (2.5) and (2.8), the theorem given in the introduction may be extended to partial differential equations of type (1.1) for which a generating function exists that has the form $E=g \exp f$ with both $f$ and $g$ suitably chosen polynomials in $t$. The generalization may be stated as follows.

TheOREM 2. Let $L(U)=0$ be a partial differential equation of type (1.1) for which a generating function $E(z, \bar{z}, t)$ exists that has one of the forms

$$
\begin{aligned}
& \text { I. } E(z, \bar{z}, t)=R(r, \theta, t) \exp P(r, \theta, t), \\
& \text { II. } E(z, \bar{z}, t)=R(r, \theta, t) \exp t P(r, \theta, t), \\
& \text { III. } E(z, \bar{z}, t)=t R(r, \theta, t) \exp t P(r, \theta, t),
\end{aligned}
$$

where

$$
\begin{aligned}
& P(r, \theta, t)=a_{0}(r, \theta)+a_{1}(r, \theta) t^{2}+\cdots+a_{m}(r, \theta) t^{2 m}, \\
& R(r, \theta, t)=b_{0}(r, \theta)+b_{1}(r, \theta) t^{2}+\cdots+b_{n}(r, \theta) t^{2 n}
\end{aligned}
$$

and where the $a_{j}(r, \theta)$ and $b_{j}(r, \theta)$ are of class $C^{\prime}$ in $r$ and $\theta$. Let $u_{p}(r, \theta)$ be the corresponding basic solutions and let $R(\partial p / \partial r)=\sum_{0}^{m+n} c_{j}(r, \theta) t^{2 j}$,

$$
\begin{gathered}
\alpha_{k}=(-1)^{k}\left(\frac{2}{r e^{i \theta}}\right)^{k} \sum_{j=k}^{m}(2 j+1) C_{j, k} a_{i} ; \\
\beta_{k}=(-1)^{k}\left(\frac{2}{r e^{i \theta}}\right)^{k} \sum_{j=k}^{n} C_{j, k} \frac{\partial b_{j}}{\partial r} ; \quad \gamma_{k}=(-1)^{k}\left(\frac{2}{r e^{i \theta}}\right)^{k} \sum_{j=k}^{n+m} C_{j, k} c_{j} .
\end{gathered}
$$

Then the recurrence relation satisfied by these basic solutions is

$$
\frac{\partial u_{p}}{\partial r}=\frac{p u_{p}}{r}+\sum_{k=0}^{n} \beta_{k} u_{p+k}+\sum_{k=0}^{n+m} \gamma_{k} u_{p+k}
$$

if $E$ has the form I; 


$$
\frac{\partial u_{p}}{\partial r}=\frac{p u_{p}}{r}+\sum_{k=0}^{n} \beta_{k} u_{p+k}+\left(\frac{2}{r e^{i \theta}}\right) \sum_{k=0}^{m} \sum_{\nu=0}^{m+n} \frac{\alpha_{k} \gamma_{\nu} u_{p+k+\nu+1}}{2 p+2 \nu+1}
$$

if $E$ has the form II; and

$$
\begin{array}{r}
\frac{\partial u_{p}}{\partial r}=\frac{p u_{p}}{r}+\left(\frac{2}{r e^{i \theta}}\right) \sum_{k=0}^{m} \sum_{\nu=0}^{n}\left(\frac{\alpha_{k} \beta_{\nu}}{2 p+2 \nu+1}\right) u_{p+k+\nu+1} \\
+\gamma_{0} u_{p}+\sum_{k=1}^{m+n}\left(\gamma_{k}-\frac{2}{r e^{i \theta}} \gamma_{k-1}\right) u_{p+k}
\end{array}
$$

if $E$ has the form III.

University of Wisconsin at Milwaukee

\section{ON GAUSS' AND TCHEBYCHEFF'S QUADRATURE FORMULAS}

\section{J. GERONIMUS}

The well known Gauss' Quadrature Formula

$$
\int_{-\infty}^{\infty} G_{k}(x) d \psi(x)=\sum_{i=1}^{n} \rho_{i}^{(n)} G_{k}\left(\xi_{i}^{(n)}\right)
$$

is valid for every polynomial $G_{k}(x)$, of degree $k \leqq 2 n-1$, the $\left\{\xi_{i}^{(n)}\right\}$ being the roots of the polynomial $P_{n}(x)$, orthogonal with respect to the distribution $d \psi(x)(i=1,2, \cdots, n ; n=1,2, \cdots) .^{1}$ If the sequence $\left\{P_{n}(x)\right\}$ is that of Tchebycheff (trigonometric) polynomials, then the Christoffel numbers $\rho_{i}^{(n)}, i=1,2, \cdots, n$, are equal, and the two quadrature formulas of Gauss and Tchebycheff coincide:

$$
\int_{-\infty}^{\infty} G_{k}(x) d \psi(x)=\rho_{n} \sum_{i=1}^{n} G_{k}\left(\xi_{i}^{(n)}\right), \quad k \leqq 2 n-1 ; n=1,2, \cdots
$$

The converse-that this is the only case of coincidence of these formulas-was proved by R. P. Bailey [1a] and, under more restrictive conditions, by Krawtchouk [1b] (cf. also [2]).2

We shall give here four distinct proofs of this statement, without imposing any restrictions on $\psi(x)$.

Received by the editors June 1, 1943.

${ }^{1} \psi(x)$ is a bounded non-decreasing function, with infinitely many points of increase, for which all moments exist: $c_{n}=\int_{-\infty}^{\infty} x^{n} d \psi(x) ; n=0,1,2, \cdots$.

${ }^{2}$ Numbers in brackets refer to the bibliography at the end of the paper. 